\section{Acral perineurioma: $A$ case report of a rare neoplasm successfully treated with autologous skin graft reconstructive surgery}

\author{
Elena Pierobon, ${ }^{1}$ Chiara Cortelazzi, ${ }^{2}$ \\ Michele Maria Dominici, ${ }^{1}$ Claudio \\ Feliciani, ${ }^{1}$ Sergio Di Nuzzo' \\ ${ }^{1}$ Section of Dermatology, Department of \\ Medicine and Surgery, University of \\ Parma; ${ }^{2}$ Section of Dermatology, \\ Department of Medicine and Surgery, \\ University of Modena and Reggio \\ Emilia, Modena, Italy
}

\begin{abstract}
Soft tissues perineurioma is a rare nerve sheath tumor that affects most of all the subcutaneous tissue. Even if it could present as a large mass, it is a benign neoplasm for which a complete surgical excision represents the gold standard treatment. Considering that it usually affects acral sites of young people, it can be challenging to perform a reconstructive surgery that allows a full functional recovery. We report the case of a woman in her 20 s presenting a perineurioma of the sole of the right foot, a nodule of about $2 \mathrm{~cm}$ of diameter that compromised the support of the foot on the ground. We performed a radical surgical excision with no recurrence after 3 years of follow up and we obtained a full functional recovery thanks to an autologous full-thickness skin graft.
\end{abstract}

\section{Case Report}

A woman in her 20 s came to our attention presenting a nodular lesion of the right sole. She referred that the lesion slowly developed after a trivial trauma without any symptom. Her medical history was completely negative up to that moment and there was no evidence of familiar conditions such as neurofibromatosis. On clinical examination, we observed a single, rounded well circumscribed nodule, with a homogeneous pink-purple color and hard on palpation (Figure 1A,B). Dermatoscopic evaluation was nonspecific and featureless (Figure $1 \mathrm{C})$.

The mass was painless, but the patient complained an initial discomfort with the pressure of the foot on the ground due to the size of the neoplasm, that was about $2 \mathrm{~cm}$ of diameter.

Histopathological analysis of a first incisional biopsy pointed to the diagnosis of perineurioma of soft tissues, even if the interpretation was more difficult because of the absence of the typical immunohistochemistry staining for the long cytoplasmic processes of perineurial cells (EMA).

We performed a complete surgical excision (Figure 2A), preserving the integrity of the plantar fascia ligament, that wasn't infiltrated by the neoplasm (Figure 2B). The loss of substance of the wound was reconstructed harvesting and applying an autologous full-thickness skin graft that healed successfully (Figure 2C,D).

After radical excision, our patient perfectly recovered with no recurrence after 3 years of clinical follow up.

\section{Discussion}

PN is a rare nerve sheath tumor that originates exclusively from perineural cells, which take part in the blood-nerve barrier surrounding peripheral nerves. ${ }^{1}$ Depending on the relationship with the nerve enveloped, PNs are classified into intraneural and extraneural ones, with different clinical presentation and etiology. ${ }^{2}$

As in our case, extraneural PN affects soft tissues and are mostly located in the subcutis. ${ }^{3}$ Typically, it occurs on acral sites and trunk of young to middle-aged adults as asymptomatic masses which can slowly reach a remarkable size. ${ }^{3}$ Extraneural $\mathrm{PN}$ is caused by deletions of chromosome $22 \mathrm{q}$ (with NF2 gene) or of chromosome 17q (with NF1 gene) so, as in other nerve sheath tumors, its onset can be facilitated in patients with neurofibromatosis. ${ }^{4}$

Nodules are usually yellow white, well circumscribed but unencapsulated. ${ }^{3}$ Clinical appearance is so aspecific that the diagnosis is based only on histopathological and immunohistochemical criteria. $^{3}$ Microscopically we observe low cellularity ${ }^{3}$ and atypical features in about $20 \%$ of subcutaneous perineuriomas, without a clinical significance. ${ }^{2}$ Most of the tumor cells are positive for the epithelial membrane antigen (EMA) immunohistochemistry, ${ }^{3}$ that highlights the cytoplasmic processes of perineurial cells. ${ }^{2}$ EMA positivity is a useful diagnostic tool, but a small subset of tumors appears to lack it, ${ }^{3}$ as in our observation.

A complete surgical excision is the treatment of choice even if the involvement of extremities and the onset among young people can make challenging to perform a radical approach and a consequent functional reconstructive surgery. Considering the weight-bearing function of the foot, a full thickness autologous skin graft is a good
Correspondence: Elena Pierobon, Section of Dermatology, Department of Medicine and Surgery, University of Parma, Via Gramsci 14, 43126 Parma, Italy.

Tel.: +39.0521.702711 - Fax: +39.0521.702959.

E-mail: elena.pierobon@studenti.unipr.it

Key words: Perineurioma, Full-thickness skin graft, Nerve sheath tumor, Acral neoplasm.

Contributions: EP participated collecting the data of the study and wrote the majority of the original draft of the paper. CC and MMD had access to all of the raw data of the study and participated in generating and gathering them. CF revised the work critically for important intellectual content. SdN participated in drafting and designing the study and analysis and interpretation of data for the work. All authors participated in writing the paper and have approved the final version.

Conflict of interest: The authors declare no potential conflict of interest.

Funding: None.

Availability of data and material: Data and materials are available by the authors.

Ethical approval and informed consent: Obtained.

Please cite this article as: Pierobon E, Cortelazzi C, Dominici MM, et al. Acral perineurioma: A case report of a rare neoplasm successfully treated with autologous skin graft reconstructive surgery. Dermatol Rep 2022;14:9093.

Received for publication: 13 May 2021. Accepted for publication: 12 July 2021.

This work is licensed under a Creative Commons Attribution-NonCommercial 4.0 International License (CC BY-NC 4.0).

${ }^{\circ}$ Copyright: the Author(s), 2022

Licensee PAGEPress, Italy

Dermatology Reports 2022; 14:9093

doi:10.4081/dr.2022.9093

treatment approach because not only it acts as wound coverage but also permits to achieve stability and durability of the reconstructed site, as in our case. ${ }^{5}$

Generally, the prognosis is good: only in few cases, about 5\% in Hornick and Fletcher's observations, a local recurrence is reported, while there is no evidence of metastasis. ${ }^{3}$

\section{Conclusions}

We report this case because it is chal- 

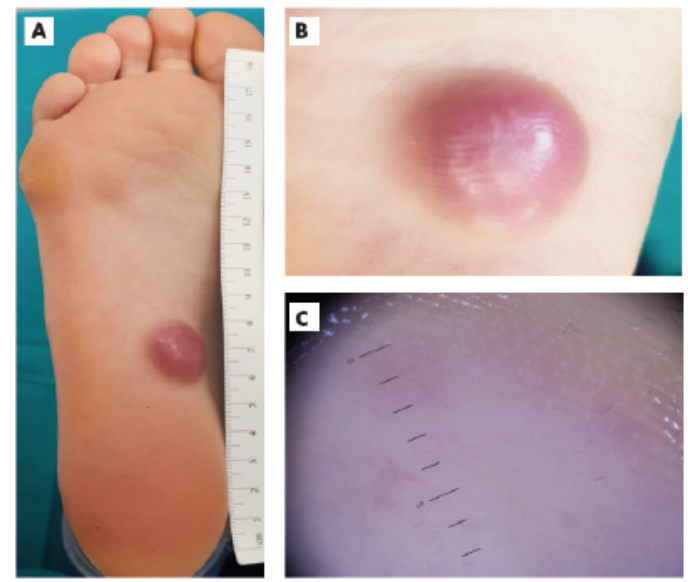

Figure 1. Clinical and dermatoscopic features of PN. A) A firm nodule of the sole of the foot of about $2 \mathrm{~cm}$ of diameter. B) Clinical detail of the well-circuscribed nodule with a homogeneous pink-purple color. C) Dermatoscopic examination didn't identify any specific vascular pattern and was featureless.
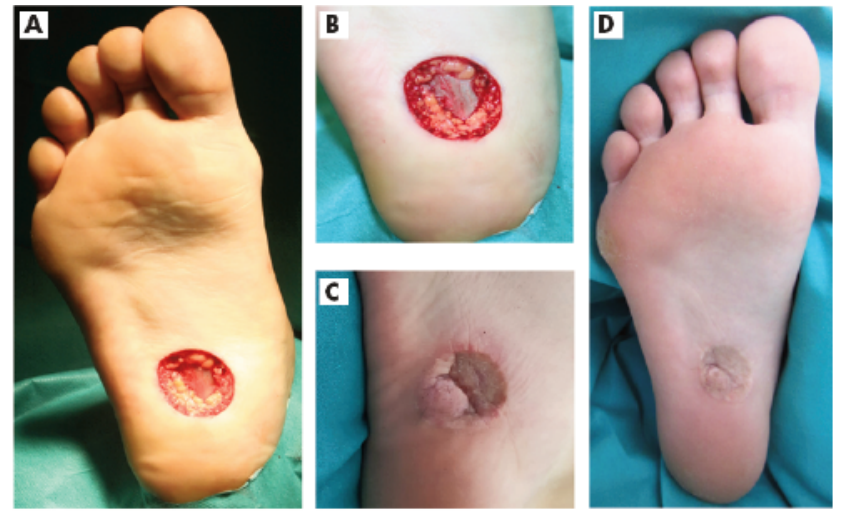

Figure 2. Surgical procedure and final outcome. A) Radical excision of the nodule of the foot with safe margins. B) Integrity of the plantar fascia ligament, at the top of which the neoplasm was located without infiltrating it. C, D) Clinical outcome of the skin graft after 3 years, perfectly healed with full recovery of the mobility function. lenging both clinically and surgically. On one hand, we would like to underline the necessity to regard some uncommon diagnoses even if the observation doesn't perfectly match all characteristics reported in literature. Extraneural $\mathrm{PN}$ is a rare entity that can be misdiagnosed because of unusual features that are not commonly reported because of the rarity of the diagnosis itself.

One the other hand, extraneural PN is a benign neoplasm with minimum risk of local recurrence and optimal prognosis after radical excision. Nevertheless, surgical removal of a tumor involving acral and functional sites could represent a challeng- ing option even when considered the treatment of choice. The consequent reconstructive procedure could be even more important than the radical excision itself and should always be performed aiming at obtaining the best functional outcome for patient.

\section{References}

1. Uerschels AK, Krogias C, Junker A et al. Modern treatment of perineuriomas: a case-series and systematic review. BMC Neurol 2020;20:1-12.
2. Rodríguez-Peralto JL, RiveiroFalkenbach E, Carrillo R. Benign cutaneous neural tumors. Semin Diagn Pathol 2013;30:45-57.

3. Hornick JL, Fletcher CDM. Soft tissue perineurioma. Am J Surg Pathol 2005;59:845-58.

4. Carter JM, Wu Y, Blessing MM et al. Recurrent genomic alterations in soft tissue perineuriomas. Am J Surg Pathol 2018;42:1708-14.

5. Yan H, Liu S, Gao W, et al. Management of degloving injuries of the foot with a defatted full-thickness skin graft. J Bone Joint Surg Am 2013;95:1675-81. 